\title{
Ultra-violet irradiation induces apoptosis via mitochondrial pathway in pancreatic cancer cells
}

\author{
TAKAHIRO YAMAUCHI ${ }^{1}$, SEIJI ADACHI ${ }^{1,2}$, ICHIRO YASUDA $^{1}$, MASANORI NAKASHIMA $^{1}$, \\ JUNJI KAWAGUCHI ${ }^{1}$, TAKASHI YOSHIOKA ${ }^{2}$, YOSHINOBU HIROSE ${ }^{3}$, \\ OSAMU KOZAWA $^{2}$ and HISATAKA MORIWAKI ${ }^{1}$ \\ Departments of ${ }^{1}$ Gastroenterology and ${ }^{2}$ Pharmacology, Gifu University Graduate \\ School of Medicine; ${ }^{3}$ Tumor Pathology, Gifu University Hospital, Gifu 501-1194, Japan
}

Received May 30, 2011; Accepted June 27, 2011

DOI: $10.3892 /$ ijo.2011.1188

\begin{abstract}
Pancreatic cancer is a highly lethal disease and gemcitabine is considered to be the standard of care for the treatment of advanced pancreatic cancer. However, the outcome of the patients treated with gemcitabine is still unstatisfactory and further development of new treatments is required. We recently found that short wavelength ultra-violet (UV-C) suppresses cell proliferation with downregulation of epidermal growth factor receptor (EGFR) in human pancreatic cancer cells, but not in normal pancreatic epithelial (PE) cells. In this study, we investigated the effect of UV-C on apoptosis in several cell lines derived from the pancreas. UV-C induced poly(ADPribose) polymerase (PARP) cleavage, which is a marker of cells undergoing apoptosis, in Panc1, MiaPaca2, KP3 and BxPC3 pancreatic cancer cells, but not in PE cells. We also observed similar effects in Hoechst 33258 staining, which shows DNA fragmentation. While p53, a tumor suppressor protein, plays a critical role in UV-C-induced cell damage, we did not observe the correlation between the sensitivity to UV-C and p53 status. Thapsigargin, an agent that promotes endoplasmic reticulum (ER) stress by depletion of lumenal calcium stores, as well as cis-diamineplatinum (II) dichloride, a classical anti-cancer drug that causes DNA damage, induced PARP cleavage even in PE cells. Moreover, UV-C-induced apoptosis in Panc1 and KP3 cells was associated with the release of cytochrome $\mathrm{c}$, indicating that it was mediated via mitochondrial pathway. Taken together, UV-C has a potent anti-cancer effect on pancreatic cancer cells without adverse effect on normal cells and it could be useful for the treatment of human pancreatic cancers.
\end{abstract}

\section{Introduction}

Pancreatic cancer is a common malignancy, ranking 13th in the incidence, and 8th as the cause of cancer related death worldwide (1). Surgical resection is the only curable treatment

Correspondence to: Dr Seiji Adachi, Department of Pharmacology, Gifu University Graduate School of Medicine, Gifu 501-1194, Japan E-mail: seijiadachi0123@gmail.com

Key words: ultra-violet, apoptosis, pancreas, cancer therapy at present but only $10-15 \%$ of patients are able to have operations at the time of diagnosis. However, it is difficult to diagnose pancreatic cancer at an early stage, even with advanced medical imagings such as computed tomography and magnetic resonance imaging. Gemcitabine has been the standard of treatment during the last decade, but the median survival time of patients treated with gemcitabine is only 5-6 months. Moreover, in clinical trials, combination of gemcitabine with certain other cytotoxic drugs, including cisplatin, oxaliplatin, capecitabine, and 5-fluorouracil have been undertaken, but all have failed to provide substantial increases in survival benefit (2). Therefore, researchers expanded their interest into the development of new treatments for inoperable pancreatic cancer.

Ultra-violet (UV) radiation from sunlight is sorted by wavelength ranges: long-wavelength UV-A $(320-400 \mathrm{~nm})$, medium-wavelength UV-B (280-320 nm) and short-wavelength UV-C (200-280 nm). UV-A and UV-B are well understood as the major carcinogenic components of sunlight (3). On the other hand, UV-C is used for studying DNA damage and cellular DNA repair process, although it does not actually exist in earth's surface since they are filtered out by the atmosphere. In general, UV-C has been practically applied for apparatus such as water sterilizer. UV radiation has many effects on skin, including inflammation, immunosuppression, and alterations in the extracellular matrix (ECM), in addition to accelerated skin aging (4). The most hazardous effect of excess UV light for humans is, however, increased risk of skin cancers (5). On the other hand, it has recently been reported that UV-C has a potential usefulness for treatment of human cancer $(6,7)$. In addition, we recently found that UV-C irradiation induces downregulation of epidermal growth factor receptor (EGFR) via phosphorylation at serine 1046/1047, which leads to the inhibition of cell proliferation in human pancreatic cancer cells (8).

Many studies demonstrated the important role of apoptosis in tumorigenesis and tumor treatment. While the completion of tumorigenesis requires a multistep process that leads to cell proliferation (9), it is essential to acquire the characteristics that protect cells from apoptosis. In addition, resistance to chemotherapy also requires the acquisition of the ability to abrogate apoptosis $(10,11)$. Among several apoptotic pathways, one possible mechanism targets mitochondria and causes mitochondrial swelling through the formation of membrane 
pores or increases the mitochondrial membrane permeability, resulting in apoptotic effectors to leak out (12). Cytochrome $\mathrm{c}$ is also released from mitochondria, and exerts a regulatory function as it precedes morphological change associated with apoptosis. In endoplasmic reticulum (ER)-mediated pathway, ER stress is caused by an accumulation of unfolded proteins in the ER lumen, and persistent ER stress can induce apoptosis (13).

In this study, we investigated the effect of UV-C on apoptosis in several types of cell lines derived from the pancreas, and demonstrated that UV-C induced apoptosis in pancreatic cancer cells but not in normal pancreatic epithelial cells. The apoptosis was mediated via mitochondria, irrespective of p53 status.

\section{Materials and methods}

Materials. Thapsigargin ( $\mathrm{Tg}$ ) and cis-diamineplatinum (II) dichloride (CDDP) were purchased from Sigma Chemical Co. (St. Louis, MO). Antibody against glyceraldehyde-3-phosphate dehydrogenase (GAPDH) was obtained from Santa Cruz Biotechnology (Santa Cruz, CA). Antibodies against PARP, cleaved PARP and p53 were purchased from Cell Signaling (Beverly, MA). The ECL Western blot detection system was purchased from GE Healthcare (Buckinghamshire, UK). Hoechest 33258 was purchased from Wako (Tokyo, Japan).

Cell culture. Primary normal human pancreatic epithelial cells (PE) were maintained in Cell Systems Corp. (CS-C) medium, purchased from DC Pharma Biomedical (Kirkland, WA). Panc1, KP3, BxPC3 cells and MiaPaca2 cells were grown in Roswell Park Memorial Institute (RPMI-1640) and Dulbecco's modified Eagle's medium (DMEM), respectively (Invitrogen, San Diego, CA). They were supplemented with $10 \%$ heat-inactivated fetal calf serum (FCS), penicillin $(100 \mathrm{U} / \mathrm{ml})$ and streptomycin $(100 \mu \mathrm{g} / \mathrm{ml})$ in a humidified $5 \% \mathrm{CO}_{2}$ incubator at $37^{\circ} \mathrm{C}$.

$U V$ - $C$ exposure. UV-C irradiation to cells was performed with UVC 500 UV Crosslinker (GE Healthcare) with 0-500 J/m² (J) UV at $254 \mathrm{~nm}$. After aspiration of the growth medium, the cells were exposed to UV-C (0 or $200 \mathrm{~J})$ and then incubated in the growth medium for the indicated periods.

Western blot analysis. The cells were lysed in lysis buffer [20 $\mathrm{mM}$ Tris- $\mathrm{HCl}$ (pH 7.5), 150 mM NaCl, 1 mM EDTA, 1 mM EGTA, $1 \%$ Triton $\mathrm{X}-100,2.5 \mathrm{mM}$ sodium pyrophosphate, $50 \mathrm{mM} \mathrm{NaF}$, $50 \mathrm{mM}$ HEPES, $1 \mathrm{mM} \mathrm{Na} \mathrm{VO}_{4}$ and $2 \mathrm{mM}$ phenylmethylsulfonyl fluoride (PMSF)] and scraped from the Petri dishes. Protein extracts were examined by Western blot analysis as previously described (14). The protein was electrophoresed and transferred onto an Immune-Blot PVDF Membrane (Bio-Rad, Hercules, CA). Membranes were blocked with $5 \%$ fat-free dry milk in phosphate-buffered saline (PBS) containing $0.1 \%$ Tween-20 (PBS-T) for $30 \mathrm{~min}$ before 16-h incubation with primary antibodies (1:1000 dilution). Peroxidase-labeled antibodies raised in goat against rabbit IgG were used as secondary antibodies (1:2000 dilution; 1-h incubation). Peroxidase activity on the membrane was visualized on X-ray film by means of the ECL Western blot detection system.

Cytochrome c releasing apoptosis assay. The detection of cytochrome $\mathrm{c}$ release was performed using Cytochrome c Releasing
(A)

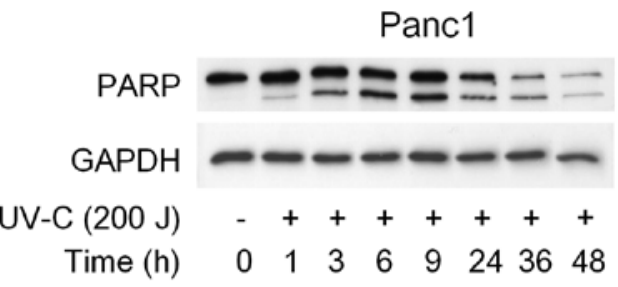

(B)

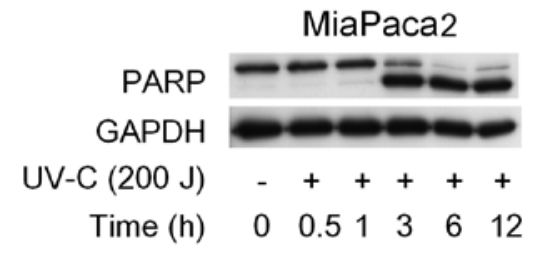

(C)

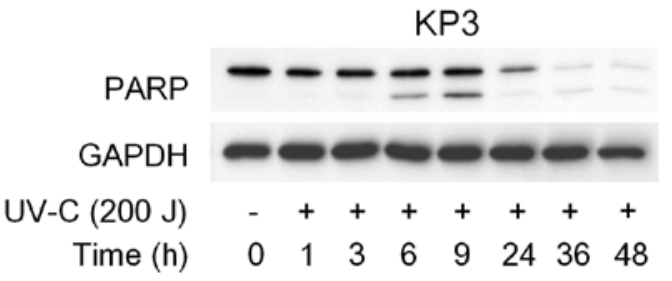

(D)

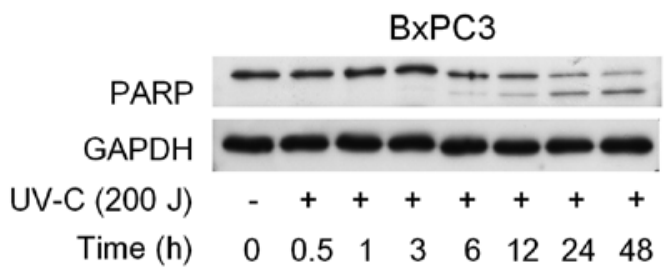

(E)

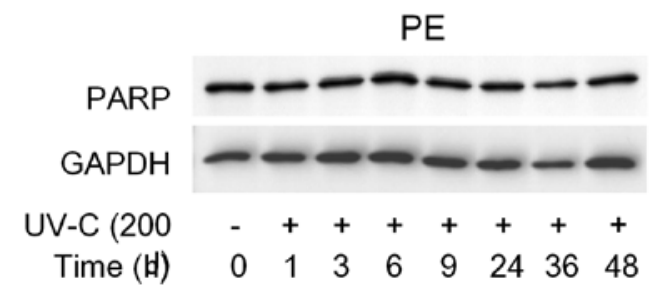

Figure 1. UV-C induced PARP cleavage in Panc1, MiaPaca2, KP3 and BxPC3, but not in PE cells. (A), MiaPaca2 (B), KP3 (C), BxPC3 (D) and PE cells (E) were exposed to UV-C at $200 \mathrm{~J}$ and further incubated for the indicated periods. Protein extracts were then harvested and examined by Western blotting using anti-PARP, anti-cleaved PARP and anti-GAPDH antibodies.

Apoptosis Assay Kit (MBL; Nagoya, Japan). In brief, the cells were exposed to UV-C and incubated for $6 \mathrm{~h}$. They were then collected by centrifugation at $600 \mathrm{x}$ g for $5 \mathrm{~min}$ at $4^{\circ} \mathrm{C}$ and washed once with cold PBS, followed by cell lysis with $1 \mathrm{X}$ Cytosol Extraction Buffer Mix containing DTT and protease inhibitors provided in the kit. They were then incubated on ice for $10 \mathrm{~min}$ and homogenized using cold dounce tissue grinder on ice 30 times. After centrifugation, the supernatant was collected as cytosolic fraction (C). Finally, the pellet was resuspended in Mitochondrial Extraction Buffer Mix containing DTT and protease inhibitors, vortexed for $10 \mathrm{sec}$ and saved as mitochondrial fraction (M).

Immunofluorescence microscopy. Immunofluorescence microscopy was performed as described previously (15). The cells grown on coverslip-bottom dishes were first exposed 


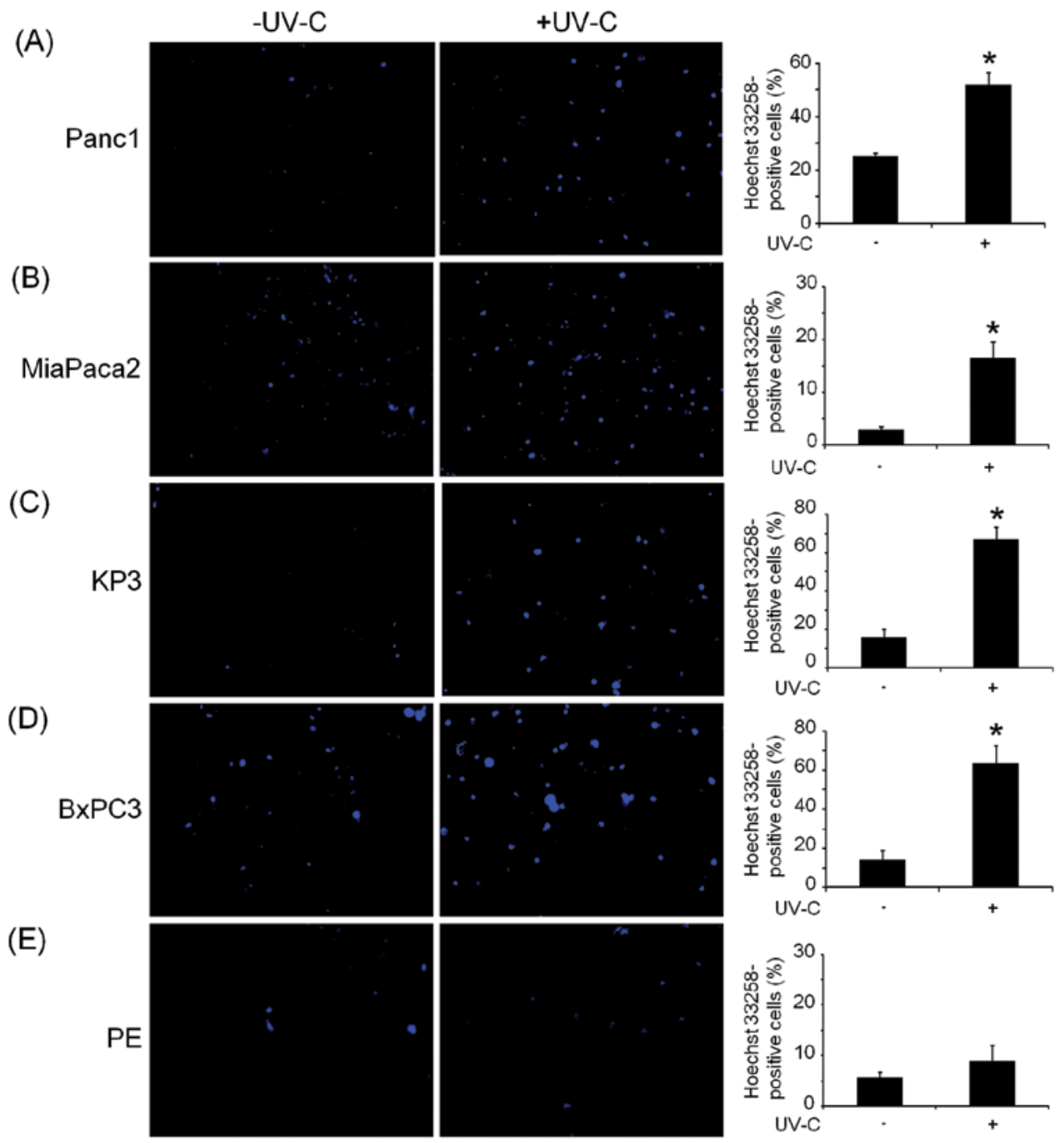

Figure 2. UV-C induced DNA fragmentation in Panc1, MiaPaca2, KP3 and BxPC3, but not in PE cells. The indicated cells were exposed to UV-C (200 J) and incubated for $24 \mathrm{~h}$. They were then treated with Hoechst 33258 (blue signal) and were examined by fluorescence microscopy. The numbers of Hoechst 33258-positive cells (apoptotic nuclei) from 5 randomly chosen fields (x40) were counted, respectively. Each value represents the mean \pm SEM of triplicate independent determinations.

to UV-C (200 J) and incubated for $6 \mathrm{~h}$ at $37^{\circ} \mathrm{C}$, followed by exposure to Hoechst 33258 for 30 min in RPMI containing 1\% bovine serum albumin (BSA). The cells were then examined by fluorescence microscopy, Biorevo (BZ-9000) (Keyence, Tokyo, Japan) according to the manufacturer's protocol.

\section{Results}

Effect of UV-C on PARP cleavage in pancreatic cancer cells and normal pancreas epithelial cells. We first performed Western blot analysis to examine the effect of UV-C on apoptosis in Panc1, MiaPaca2, KP3 and BxPC3 pancreatic cancer cells, in comparison with a human normal pancreatic epithelial cell line, PE cells. PARP helps cells to maintain their viability, and cleavage of PARP induces apoptosis (16), indicating that cleaved PARP is observed in the cells undergoing apoptosis. We found that UV-C induced cleavage of PARP in Panc1, MiaPaca2, KP3 and BxPC3 cells (Fig. 1A-D). In these cells, cleaved PARP were observed at 3-6 h after exposure to UV-C (200 J). Interestingly, UV-C had little effect on PARP cleavage in PE cells even $48 \mathrm{~h}$ after exposure to UV-C (Fig. 1E). We previously reported that $\mathrm{UV}-\mathrm{C}$ causes a marked inhibition in BrdU incorporation in Panc1 and KP3 cells, but it caused less reduction in PE cells, suggesting that UV-C has a potent antiproliferative effect on Panc1 and KP3 pancreatic cancer cells, compared with PE normal pancreatic epithelial cells (8). In case of apoptosis, there are also differences in the sensitivity to $\mathrm{UV}-\mathrm{C}$ between pancreatic cancer cells and PE cells.

Effect of $U V-C$ on DNA fragmentation in pancreatic cancer cells and normal pancreas epithelial cells. Apoptosis is a form of programmed cell death characterized by cytoplasmic condensation, leading to DNA strand breaks, which is detected at the very early stages of apoptosis (17). Such fragments are easily detected by the Hoechst 33258 staining allowing for early detection of apoptosis in cultured cells. Since we observed that UV-C caused PARP cleavage in Panc1, MiaPaca2, KP3 and BxPC3 cells, but not in PE cells, we next examined the effect of UV-C on DNA fragmentation utilizing Hoechst 33258. As expected, we observed that Hoechst 33258-positive apoptotic cells were significantly increased in Panc1, MiaPaca2, KP3 and BxPC3 cells (Fig. 2A-D), but not in PE cells (Fig. 2E). 


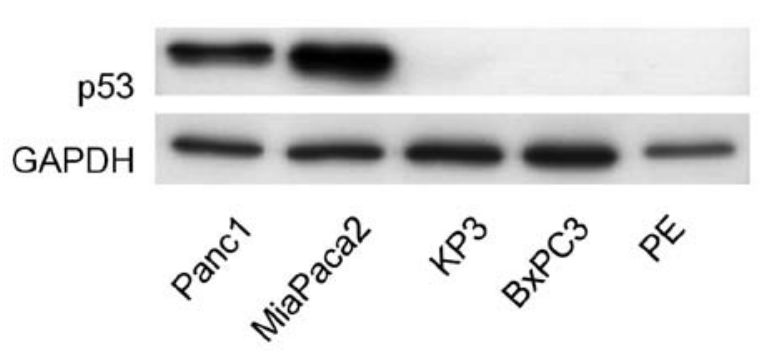

Figure 3. (A) Expression level of p53 protein in Panc1, MiaPaca2, KP3, BxPC3 and PE cells. The indicated cells were harvested and protein extract (5 $\mu \mathrm{g}$ each) were applied to Western blotting using anti-p53 and anti-GAPDH antibodies.

Taken together with the results shown in Fig. 1, UV-C selectively induced apoptosis in pancreatic cancer cells.

The status of p53 in pancreatic cancer cells and normal pancreatic epithelial cells. The tumor suppressor p53 is activated by several forms of cellular stress, including UV-C, that induce p53-mediated cell cycle arrest and/or apoptosis (3). Therefore, we next examined the expression level of p53 in unstimulated pancreatic cancer cells. As shown in Fig. 3, p53 was highly expressed in Panc1 and MiaPaca2 cells, while faint bands was seen in KP3, BxPC3 and PE cells. We also examined the effect of UV-C on p53, but found that UV-C did not affect the expression level of p53 in these cells (data not shown). Moreover, UV-C induced phosphorylation of p53 at Ser6, 9, 15, 20, 37, 46 and 392 only in Panc1 and MiaPaca 2 cells, but not in KP3, BxPC3 and PE cells (data not shown). These results suggest that p53 status might not be correlated to the UV-C sensitivity of the cells derived from the pancreas.

Effects of Tg and CDDP on PARP cleavage in Pancl, KP3 and $P E$ cells. Thapsigargin, a non-competitive inhibitor of $\mathrm{Ca}^{2+}$ ATPase on endoplasmic reticulum (ER), causes ER stress which can induce apoptosis (13). CDDP is a platinum-based chemotherapy drug used to treat various types of cancers and causes crosslinking of DNA which eventually leads apoptosis (18). We next compared UV-C to Tg or CDDP on PARP cleavage in Panc1, KP3 and PE cells. As depicted in Fig. 4, while UV-C induced PARP cleavage in Panc1 and KP3 cells but not in PE cells, Tg as well as CDDP induced PARP cleavage in all cell lines. These results indicate that the sensitivity to Tg and CDDP does not differ among the cell lines.
(A) Panc1
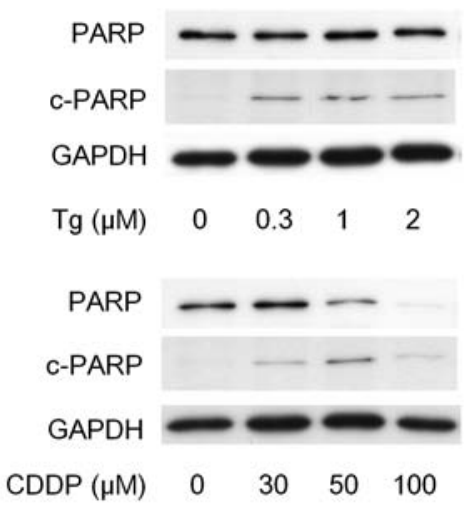

(B) KP3

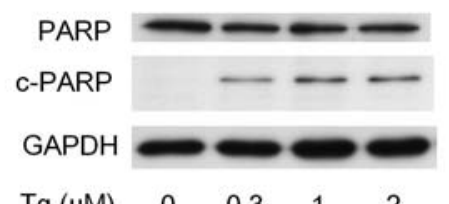

$\operatorname{Tg}(\mu \mathrm{M}) \quad 0 \quad 0.3 \quad 1 \quad 2$

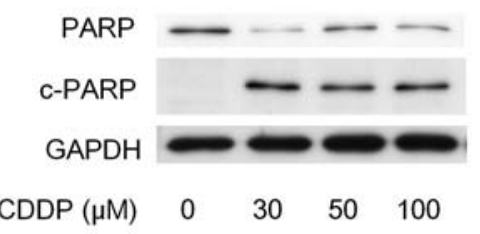

(C) PE

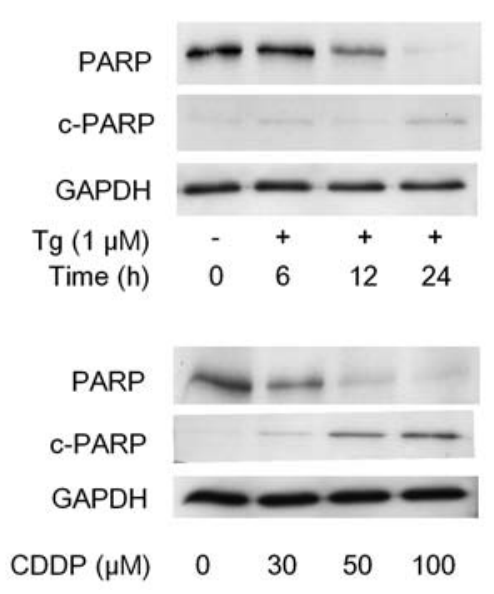

Figure 4. Both Tg and CDDP cause PARP cleavage in PE cells as well as Panc1 and KP3 cells. The indicated cells (Panc1, KP3 and PE) were treated with Thapsigargin (Tg; 0, 0.3, 1 and $2 \mu \mathrm{M}$ ) or cis-diamineplatinum (II) dichloride (CDDP; 0, 30, 50 and $100 \mu \mathrm{M}$ ) for $24 \mathrm{~h}$. PE cells were treated with $1 \mu \mathrm{M}$ of Tg for the indicated times. Protein extracts were then harvested and examined by Western blotting using anti-PARP, anti-cleaved PARP and anti-GAPDH antibodies. 
(A)

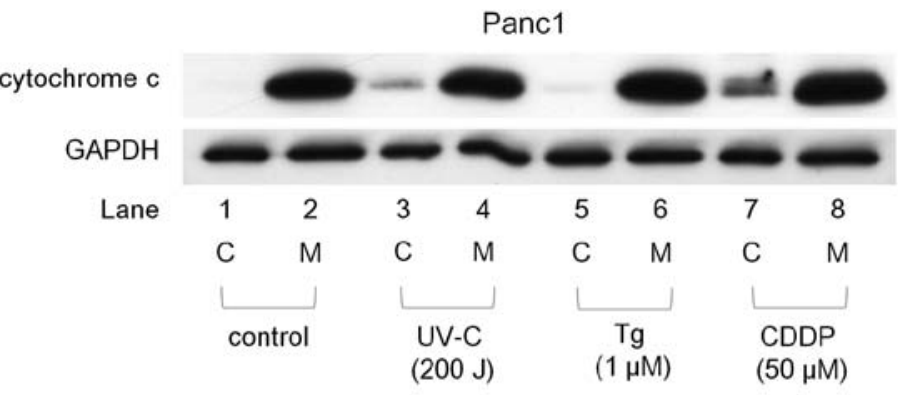

(B)

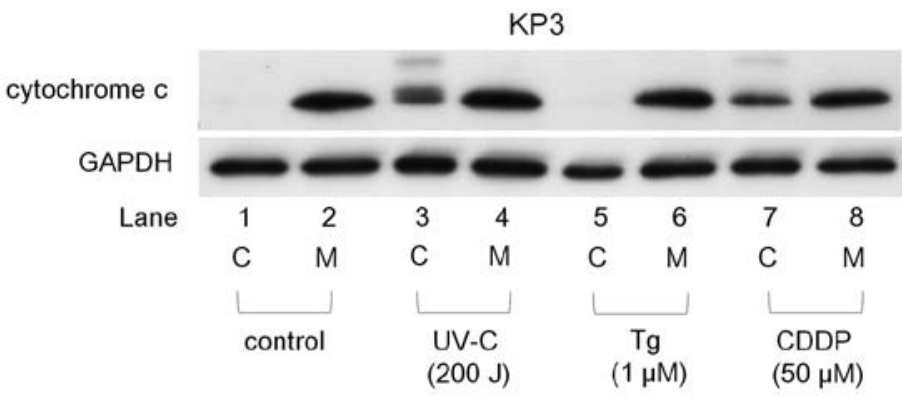

(C)

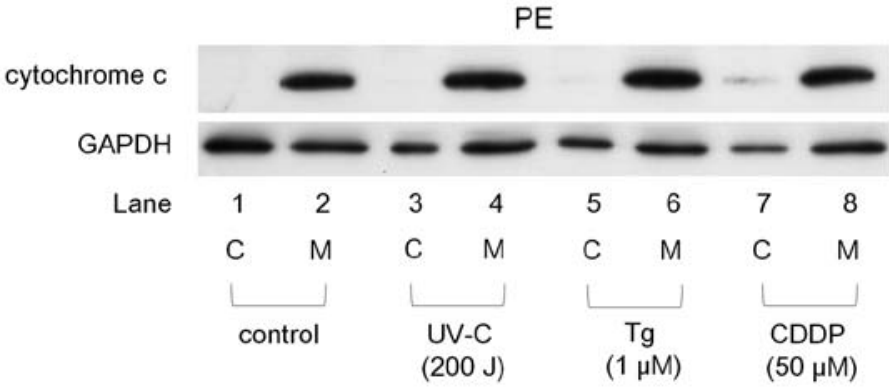

Figure 5. UV-C induces release of cytochrome c in Panc1 and KP3 cells, but not in PE cells. The indicated cells (lanes 3 and 4 ) were exposed to UV-C (200 J) and incubated for $24 \mathrm{~h}$. The other cells were treated with $1 \mu \mathrm{M}$ of Thapsigargin (Tg; lanes 5 and 6 ) or $50 \mu \mathrm{M}$ of cis-diamineplatinum (II) dichloride (CDDP; lanes 7 and 8) for $24 \mathrm{~h}$. The detection of cytochrome c release was performed using Cytochrome c Releasing Apoptosis Assay Kit as manufacturer's protocol. Protein extracts were then harvested and examined by Western blotting using anti-cytochrome c and anti-GAPDH antibodies. C, cytosolic fraction; and M, mitochondrial fraction.

Effect of $U V$-C on cytochrome c release in Pancl, KP3 and $P E$ cells. We next performed cytochrome c releasing assay to examine whether UV-C-induced apoptosis is mediated via mitochondria pathway. In untreated cells, the bands of cytochrome c were detected only in mitochondrial fraction $(\mathrm{M})$, but not in cytosol fraction (C) (Fig. 5A, B and C; lanes 1-2, respectively). Whereas $\mathrm{Tg}$ did not cause the release of cytochrome $\mathrm{c}$ in Panc1, Kp3 and PE cells (Fig. 5A, B and C; lane 5, respectively), CDDP clearly induced cytochrome c release from mitochondria to cytosol (Fig. 5A, B and C; lane 7, respectively). As for UV-C, it caused the release of cytochrome $\mathrm{c}$ in Panc1 and KP3, but not in PE cells (Fig. 5A, B and C; lanes 3-4, respectively), thus suggesting that UV-C-induced apoptosis is mediated via mitochondrial pathway.

\section{Discussion}

In this study, we shed light on the usefulness of short wavelength ultra-violet (UV-C) in lethal pancreatic cancer, because present chemotherapy based on gemcitabine treatment is not yet satisfactory and we need to mark a dramatic shift against pancreatic cancer. We demonstrated that UV-C induced apoptosis via mito- chondrial pathway in several types of pancreatic cancer cells, but not in normal pancreatic epithelial cells (Figs. 1, 2 and 5). p53 protein is a central factor in cellular stress responses and acts as an essential tumor suppressor (3). Upon activation, p53 determines the fate of the cell based on the severity of the damage. Thus, it can inhibit cell cycle progression and repair damage directly (3). In case of irreparable damage, p53 protein induces apoptosis $(19,20)$. In addition, $>50 \%$ of human tumors have been reported to have p53 mutations, which make p53 function impaired (3). p53 is reportedly mutated in Panc1 and MiaPaca2 cells (21), and we found that UV-C induced phosphorylation of p53 at several Ser residues including Ser 46, which is important for induction of p53-regulated apoptosis-inducing protein 1 (22). However, we did not detect 553 protein in KP3 and BxPC 3 cells (Fig. 3). In addition, UV-C did not induce its expression in these cells (data not shown). Based on these results, it seems unlikely that the sensitivity to UV-C in the induction of apoptosis is due to the p53 status. Although PE cells were less sensitive to UV-C as compared to pancreatic cancer cells, both of Tg as an ER stress inducer, and CDDP as a classical DNA damaging agent, actually caused apoptosis even in PE cells (Fig. 4). Therefore, our findings indicate that UV-C has potent anti-cancer effect 
without adverse effect on normal pancreatic cells. We recently reported that UV-C induces activation of p44/p42 mitogen activated protein kinase (MAPK), stress-activated protein kinase/c-Jun-N-terminal kinase (SAPK/JNK), p38 MAPK and Akt in pancreatic cancer cells as well as PE cells and that p38 MAPK plays a critical role in the downregulation of EGFR (8). However, inhibitor or siRNA experiments did not reveal that these kinases are involved in UV-C-induced apoptosis (data not shown), thus indicating that the mechanism of UV-C-induced EGFR downregulation differs from that of UV-C-induced apoptosis.

While researchers use UV-C as a tool for DNA damage, it has previously been reported that UV-C causes cell cycle arrest in G1 phase (7). In addition, we recently found that UV-C irradiation leads to the inhibition of cell proliferation, concurrently with downregulation of EGFR via phosphorylation at serine 1046/1047 in human pancreatic cancer cells (8). Taken together with the present study, our findings might provide a new possible aspect of pancreatic cancer therapy, although further investigation is required to develop devices that supply UV-C efficiently into human pancreatic cancer tissue, for example with endoscopic/laparoscopic approach.

In conclusion, our results strongly suggest that UV-C induces apoptosis via mitochondrial pathway in pancreatic cancer cells. The present study on UV-C could provide a novel promising therapeutic strategy.

\section{Acknowledgments}

We are very grateful to Ms. Yoko Kawamura for her skillful technical assistance. This work was supported in part by a Grant-in-Aid for Scientific Research (22790639 to S.A.) from the Ministry of Education, Science, Sports and Culture of Japan, and a grant by Yokoyama Foundation for Clinical Pharmacology.

\section{References}

1. Parkin DM, Bray F, Ferlay J and Pisani P: Global cancer statistics, 2002. CA Cancer J Clin 55: 74-108, 2005.

2. Sanchez SE and Trevino JG: Current adjuvant and targeted therapies for pancreatic adenocarcinoma. Curr Med Chem 15: 1674-1683, 2008.
3. Latonen L and Laiho M: Cellular UV damage responses-functions of tumor suppressor p53. Biochim Biophys Acta 1755: 71-89, 2005.

4. Norval M: Effects of solar radiation on the human immune system. J Photochem Photobiol 63: 28-40, 2001.

5. De Gruijl FR: Skin cancer and solar UV radiation. Eur J Cancer 35: 2003-2009, 1999.

6. Zwang Y and Yarden Y: p38 MAP kinase mediates stress-induced internalization of EGFR: implications for cancer chemotherapy. EMBO J 25: 4195-4206, 2006.

7. Olsen BB, Neves-Petersen MT, Klitgaard S, Issinger OG and Petersen SB: UV light blocks EGFR signalling in human cancer cell lines. Int J Oncol 30: 181-185, 2007.

8. Yamauchi T, Adachi S, Yasuda I, et al: UV-C irradiation induces downregulation of EGF receptor via phosphorylation at serine 1046/1047 in human pancreatic cancer cells. Radiat Res (In press).

9. Felsher DW: Oncogene addiction versus oncogene amnesia: perhaps more than just a bad habit? Cancer Res 68: 3081-3086, 2008.

10. Pedraza-Farina LG: Mechanisms of oncogenic cooperation in cancer initiation and metastasis. Yale J Biol Med 79: 95-103, 2006.

11. Mehlen P and Puisieux A: Metastasis: a question of life or death. Nat Rev Cancer 6: 449-458, 2006

12. Brune B: Nitric oxide: NO apoptosis or turning it ON? Cell Death Differ 10: 864-869, 2003.

13. Verfaillie T, Garg AD and Agostinis P: Targeting ER stress induced apoptosis and inflammation in cancer. Cancer Lett (In press).

14. Adachi S, Nagao T, To S, et al: (-)-Epigallocatechin gallate causes internalization of the epidermal growth factor receptor in human colon cancer cells. Carcinogenesis 29: 1986-1993, 2008.

15. Adachi S, Shimizu M, Shirakami Y, et al: (-)-Epigallocatechin gallate downregulates EGF receptor via phosphorylation at Ser1046/1047 by p38 MAPK in colon cancer cells. Carcinogenesis 30: 1544-1552, 2009.

16. Oliver FJ, De la Rubia G, Rolli V, Ruiz-Ruiz MC, De Murcia G and Murcia JM: Importance of poly(ADP-ribose) polymerase and its cleavage in apoptosis. Lesson from an uncleavable mutant. J Biol Chem 273: 33533-33539, 1998.

17. Darzynkiewicz Z, Bruno S, Del Bino G, et al: Features of apoptotic cells measured by flow cytometry. Cytometry 13: 795-808, 1992.

18. Usanova S, Piee-Staffa A, Sied U, et al: Cisplatin sensitivity of testis tumour cells is due to deficiency in interstrand-crosslink repair and low ERCC1-XPF expression. Mol Cancer 9: 248, 2010.

19. Prives C and Hall PA: The p53 pathway. J Pathol 187: 112-126, 1999.

20. Vousden $\mathrm{KH}$ and Lu X: Live or let die: the cell's response to p53. Nat Rev Cancer 2: 594-604, 2002.

21. Butz J, Wickstrom E and Edwards J: Characterization of mutations and loss of heterozygosity of p 53 and K-ras 2 in pancreatic cancer cell lines by immobilized polymerase chain reaction. BMC Biotechnol 3: 11, 2003.

22. Oda K, Arakawa H, Tanaka T, et al: p53AIP1, a potential mediator of p53-dependent apoptosis, and its regulation by Ser-46phosphorylated p53. Cell 102: 849-862, 2000. 